\title{
The efficacy and safety of drug-eluting balloons for the treatment of in-stent restenosis as com- pared with drug-eluting stents and with conven- tional balloon angioplasty
}

Pyung Chun Oh, Soon Yong Suh, Woong Chol Kang, Kyounghoon Lee, Seung Hwan Han, Taehoon Ahn, and Eak Kyun Shin

Division of Cardiology, Department of Internal Medicine, Gachon University Gil Medical Center, Incheon, Korea
Background/Aims: Treatment of coronary in-stent restenosis (ISR) is still associated with a high incidence of recurrence. We aimed to compare the efficacy and safety of drug-eluting balloons (DEB) for the treatment of ISR as compared with conventional balloon angioplasty (BA) and drug-eluting stents (DES).

Methods: Between January 2006 and May 2012 a total of 177 patients (188 lesions, $64.1 \pm 11.7$ years old) who underwent percutaneous coronary intervention for ISR were retrospectively enrolled. Clinical outcomes were compared between patients treated with DEB ( $\mathrm{n}=58,32.8 \%)$, conventional BA $(\mathrm{n}=65,36.7 \%)$, or DES $(\mathrm{n}=54$, $30.5 \%)$. The primary end point was a major adverse cardiac event (MACE), defined as a composite of cardiac death, myocardial infarction, and target lesion revascularization (TLR).

Results: Baseline characteristics were not different except for a history of previous MI, which was more frequent in patients treated by conventional BA or DES than in patients treated by DEB ( $40.0 \%$ vs. $48.1 \%$ vs. $17.2 \%$, respectively, $p=0.002$ ). The total incidences of MACEs were $10.7 \%, 7.4 \%$, and $15.4 \%$ in patients treated by DEB, DES, or conventional BA, respectively ( $p>0.05)$. TLR was more frequent in patients treated by conventional BA than in patients treated by DEB or DES, but this was not statistically significant (10.8\% vs. $6.9 \%$ vs. $3.7 \%, p>0.05$ between all group pairs, respectively).

Conclusions: This study showed that percutaneous coronary intervention using DEB might be a feasible alternative to conventional BA or DES implantation for treatment of coronary ISR. Further large-scaled, randomized study assessing long-term clinical and angiographic outcomes will be needed.

Keywords: Drug eluting stent; Coronary restenosis

\section{INTRODUCTION}

In-stent restenosis (ISR) after stent implantation remains a frequent problem in coronary interventional therapy. Although drug-eluting stents (DES) significantly reduce the rates of restenosis and target lesion revascularization (TLR) [1,2], the increasing use of DES in complex patients and lesions with high restenotic po- 
tentials has resulted in double-digit restenosis rates $[3,4]$.

The best treatment strategy for ISR has not been established and its identification remains challenging, although several treatment options, such as, conventional balloon angioplasty (BA), intracoronary brachytherapy, and the implantation of an additional stent are available. In recent years, the local delivery of an antiproliferative drug from drug-eluting balloons (DEB) has emerged as a potential alternative for the treatment of ISR. However, although previous clinical trials had reported DEB might be safe and effective for the treatment of ISR [5-8], its role in the management of ISR has not been comprehensively established. Therefore, the aim of the study was to assess the safety and efficacy of DEB as compared with conventional BA or the implantation of a second DES for the treatment of ISR.

\section{METHODS}

The retrospective study was performed using the Gachon University Gil Medical Center percutaneous coronary intervention (PCI) database. Patients who underwent PCI in ISR lesions were selected and constituted the eligible 177 patients (188 lesions) between January 2006 and May 2012. Inclusion criteria were an ISR in bare-metal or DES, reference vessel diameter of 2.5 to $3.5 \mathrm{~mm}$, and lesion length $<30 \mathrm{~mm}$. Exclusion criteria were thrombus within the target vessel, clinically significant calcification of the target lesion, bifurcation lesion, total coronary artery occlusion, contraindication to dual antiplatelet therapy. The patients were divided into three treatment modality groups, as follows; the BA group (n $=65,36.7 \%$ ), members of which were treated by conventional BA using an uncoated balloon catheter, the DES group ( $\mathrm{n}=54,30.5 \%)$, members were implanted with a second DES, and the DEB group ( $\mathrm{n}=58,32.8 \%)$, treated by BA using a DEB. The treatment modality for ISR was decided by the interventional cardiologist. All ISR lesions were predilated with an uncoated balloon catheter according to the size of the restenotic stent. In the DEB group, a paclitaxel-eluting balloon was used (SeQuent Please balloon catheter, B. Braun Melsungen, Berlin, Germany) and the diameter of DEB had to be at least the diameter of the pre-dilatation balloon. Patients received heparin to an activated clotting time of 200 to 250 seconds. Dual antiplatelet therapy with acetylsalicylic acid $100 \mathrm{mg}$ and clopidogrel $75 \mathrm{mg}$ once daily was prescribed for at least 6 months. Clinical and demographic characteristics, angiographic and procedural data, and clinical follow-up outcomes were reviewed from medical records. ISR morphology was classified according to the Mehran classification [9]. The primary end point was defined as the occurrence of a major adverse cardiovascular event (MACEs), which included cardiac death, myocardial infarction (MI), and TLR. Stent thrombosis was defined as described by the Academic Research Consortium as 'definite' when acute coronary syndrome was present with angiographic or autopsy evidence of thrombus or occlusion, or as 'probable' when unexplained death occurred within 30 days of the procedure or acute MI involving the target vessel occurred without angiographic confirmation [10].

\section{Statistical analysis}

All continuous variables are presented as mean \pm standard deviations for normally distributed data or as median (interquartile ranges) for skewed data. Categorical variables are described using absolute and relative (percentage) frequencies. The three groups were compared with respect to clinical and demographic characteristics and procedural data using the unpaired $t$ test (for two groups) or using one-way analysis of variance (for three groups) for continuous variables or using Pearson chisquare test for categorical variables. $p$ values of less than 0.05 were considered statistically significant, and the analysis was performed using SPSS version 18.0 (SPSS Inc., Chicago, IL, USA).

\section{RESULTS}

\section{Baseline characteristics}

Table 1 showed the clinical and demographic characteristics of the three groups. Baseline characteristics were well balanced except for a history of previous MI, which was more common in the BA and DES groups than in the DEB group ( $40.0 \%$ vs. $48.1 \%$ vs. $17.2 \%$; $p=0.002$, respectively). The proportion of diabetic patient was similar in the three groups ( $40 \%$ in each group). There was no significant difference of the proportion of acute coronary syndrome as the clinical presentation of ISR in 
Table 1. Baseline characteristics

\begin{tabular}{|c|c|c|c|}
\hline Characterisctic & $\operatorname{DEB}(\mathrm{n}=58)$ & $\operatorname{DES}(\mathrm{n}=54)$ & $\mathrm{BA}(\mathrm{n}=65)$ \\
\hline Age, yr & $63.3 \pm 11.8$ & $64.8 \pm 10.8$ & $64.2 \pm 12.7$ \\
\hline Male sex & $36(62.1)$ & $40(74.1)$ & $49(75 \cdot 4)$ \\
\hline Diabetes & $25(43.1)$ & $20(37.0)$ & $27(41.5)$ \\
\hline Hypertension & $36(62.1)$ & $26(48.1)$ & $44(67 \cdot 7)$ \\
\hline Current smoker & $8(13.8)$ & $12(22.2)$ & $17(26.2)$ \\
\hline Previous myocardial infarction $^{\mathrm{a}}$ & $10(17.2)$ & $26(48.1)$ & $26(40.0)$ \\
\hline \multicolumn{4}{|l|}{ Clinical presentation of ISR } \\
\hline Stable angina & $46(79 \cdot 3)$ & $40(74.0)$ & $52(80.0)$ \\
\hline Unstable angina & $7(12.1)$ & $10(18.5)$ & $8(12.3)$ \\
\hline Acute myocardial infarction & $5(8.6)$ & $4(7.4)$ & $5(7.7)$ \\
\hline
\end{tabular}

Values are presented as mean \pm SD or number (\%).

DEB, drug-eluting balloon; DES, drug-eluting stent; BA, conventional balloon angioplasty; ISR, in-stent restenosis. ${ }^{a} p=0.002$ between three groups.

\section{Table 2. Lesion characteristics}

\begin{tabular}{|c|c|c|c|}
\hline Characteristic & DEB & DES & $\mathrm{BA}$ \\
\hline No. of lesions & 67 & 54 & 67 \\
\hline \multicolumn{4}{|l|}{ Target vessel } \\
\hline Left main & $2(3.0)$ & o & $2(3.0)$ \\
\hline Left anterior descending & $31(46.3)$ & $27(50.0)$ & $31(46.3)$ \\
\hline Left circumflex & $10(14 \cdot 9)$ & $7(13.0)$ & $15(22.4)$ \\
\hline Right coronary artery & $24(35.8)$ & $20(37.0)$ & $19(28.4)$ \\
\hline \multicolumn{4}{|l|}{ Previous stent } \\
\hline Stent diameter, $\mathrm{mm}$ & $3.0 \pm 0.4$ & $3.1 \pm 0.4$ & $3.0 \pm 0.4$ \\
\hline Stent length, mm & $22.5 \pm 6.3$ & $21.7 \pm 5 \cdot 5$ & $24.2 \pm 7.4$ \\
\hline \multicolumn{4}{|l|}{ Stent type } \\
\hline Bare-metal stent ${ }^{\mathrm{a}}$ & $14(20.9)$ & $24(44 \cdot 4)$ & $12(17 \cdot 9)$ \\
\hline Drug-eluting stent ${ }^{\mathrm{a}}$ & $47(70.1)$ & $24(44 \cdot 4)$ & $52(77.6)$ \\
\hline No available & $6(9.0)$ & $6(11.1)$ & $3(4 \cdot 5)$ \\
\hline \multicolumn{4}{|l|}{ In-stent restenosis patterns ${ }^{b}$} \\
\hline $\mathrm{I}$ (focal) & $40(59.7)$ & $32(60.4)$ & $38(58.5)$ \\
\hline II (diffuse) ${ }^{\mathrm{a}}$ & $23(34 \cdot 3)$ & $6(11.3)$ & $13(20.0)$ \\
\hline III (proliferative) & 0 & $4(7 \cdot 5)$ & $8(12.3)$ \\
\hline IV (occlusive) ${ }^{\mathrm{a}}$ & $4(6.0)$ & $11(20.8)$ & $6(9.2)$ \\
\hline
\end{tabular}

Values are presented as number (\%) or mean \pm SD.

DEB, drug-eluting balloon; DES, drug-eluting stent; BA, conventional balloon angioplasty.

${ }^{a} p<0.05$ between three groups.

${ }^{b}$ Pattern of in-stent restenosis according to the Mehran classification [9].

the DEB, DES, and BA groups (20.7\% vs. $25.9 \%$ vs. $20.0 \%$; $p=0.707$, respectively)

\section{Angiographic and procedural data}

Table 2 summarized angiographic data. Vessels treated vessel for ISR and previous stent diameters and lengths 
Table 3. Clinical outcomes during follow-up

\begin{tabular}{|c|c|c|c|c|c|c|}
\hline \multirow{2}{*}{ Variable } & \multirow{2}{*}{$\operatorname{DEB}(\mathrm{n}=58)$} & \multirow{2}{*}{$\operatorname{DES}(\mathrm{n}=54)$} & \multirow{2}{*}{$\mathrm{BA}(\mathrm{n}=65)$} & \multicolumn{3}{|c|}{$p$-value } \\
\hline & & & & DEB vs. DES & DEB vs. BA & DES vs. BA \\
\hline Follow-up period, mon & $17.2 \pm 8.7$ & $16.9 \pm 13.4$ & $15.4 \pm 11.6$ & 0.889 & 0.352 & 0.529 \\
\hline MACEs & $5(8.6)$ & $5(9 \cdot 3)$ & $9(13.8)$ & 1.000 & 0.407 & 0.571 \\
\hline Cardiac death & $1(1.7)$ & $2(3.7)$ & $2(4.1)$ & 0.608 & 1.000 & 1.000 \\
\hline Myocardial infarction & o & $2(1.9)$ & o & 0.230 & - & 0.204 \\
\hline TLR & $4(6.9)$ & $1(3.7)$ & $7(10.8)$ & 0.365 & 0.537 & 0.070 \\
\hline Stent thrombosis & o & $2(3.7)$ & $1(1.5)$ & 0.230 & 1.000 & 0.590 \\
\hline
\end{tabular}

Values are presented as mean \pm SD or number (\%).

DEB, drug-eluting balloon; DES, drug-eluting stent; BA, conventional balloon angioplasty; MACE, major adverse cardiac event; TLR, target lesion revascularization.

in ISR lesions were similar in the three groups. However, type of previous stent was significantly different. For treatment of ISR after bare-metal stent (BMS) implantation, DES was more frequently used than BA or DEB ( $48.0 \%$ vs. $35.0 \%$ vs. $24.0 \%$; $p=0.001$, respectively). Regarding ISR morphology, although proportions wth the focal ISR pattern (type I) were similar in the three groups (about 60\%), the DEB group had a greater proportion with the diffuse ISR pattern (type II) than the BA or DES groups (34.3\% vs. $20.0 \%$ vs. $11.3 \% ; p=0.009$, respectively). On the other hand, the occlusive ISR pattern (type IV) was more frequent in the DES group than in the BA or DEB groups (20.8\% vs. $9.2 \%$ vs. $6.0 \%$; $p=$ 0.032 , respectively).

In the DEB group, mean diameter and length of the paclitaxel-eluting balloon catheter were $3.0 \pm 0.3$ and 21.8 $\pm 4.4 \mathrm{~mm}$, respectively. Of the 69 lesions treated by DEB, 6 lesions (8.7\%) required additional BMS implantation for treating DEB-induced dissections.

\section{Clinical outcomes}

Table 3 summarizes clinical outcomes during follow-up. The mean overall follow-up period was $16.3 \pm 11.2$ months and was not significantly different between the three groups. The total incidences of MACEs were 10.7\%, 7.4\%, and $15.4 \%$ in the DEB, DES, and BA groups, respectively ( $p>0.05$ between all group pairs). Regardless of the type of used stent, there were no significant difference of the incidence of MACEs in the DEB, DES, and BA groups. There was no statistical difference in TLR between groups. Rates of stent thrombosis were not significantly different.

\section{DISCUSSION}

In this study, we evaluated the efficacy and safety of DEB with respect to clinical outcomes for the treatment of ISR as compared with DES and conventional BA. Our results show no significant difference between these three modalities with respect to MACE rate, and suggest DEB can be a feasible and effective option for the treatment of ISR.

The optimal treatment for ISR remains to be determined. Conventional BA for ISR is limited by high rates of repeat restenosis (39\% to $45 \%$ ) [11,12], and BMS implantation has similar results to conventional BA for the treatment of ISR [11]. The implantation of an additional DES, that is, a sirolimus- or paclitaxel-eluting stent, in ISR lesions resulted in rates of restenosis of $14 \%$ to $22 \%$ $[13,14]$. However, these stent-in-stent approaches raise concerns about the long-term implications of several stent layers in the native coronary artery wall regarding: (1) an increased risk of late stent thrombosis due to the chronic inflammation of non-resorbable polymers or delayed, incomplete endothelialization; (2) suboptimal stent geometry or insufficient stent expansion, which could cause recurrent restenosis; and (3) limited treatment options in case of recurrent restenosis after second stent implantation [6].

DEB offers a potential alternative for the treatment of ISR, and can provide several advantages as compared with second stent implantation as follows: (1) the homogenous delivery of antiproliferative medication; (2) the temporary release of high concentrations of medication, which can lead to a slight enhancement of long- 
term healing; (3) the absence of multiple layers of stents and polymers, which can decrease chronic inflammation and late stent thrombosis; and (4) a significant reduction in dual antiplatelet therapy $[15,16]$.

The Treatment of In-Stent Restenosis using Paclitaxel Coated Balloon Catheters (PACCOCATH ISR I and PACCOCATH ISR II) trials demonstrated that patients treated with paclitaxel-coated balloon had better angiographic results (less late luminal loss and binary restenosis) and improved clinical outcomes at 12 months and more long-term follow-up, as compared with patients treated with an uncoated balloon $[5,7,17]$.

In the Paclitaxel-Eluting Balloon Catheter in Coronary Artery Disease (PEPCAD II ISR) study, the use of a paclitaxel-eluting balloon for the treatment of BMS ISR significantly reduced late lumen loss and binary restenosis rate at 6-month angiographic follow-up visits and improved 1-year event-free survival as compared with paclitaxel-eluting stents [18]. In addition, the PEPCAD DES trial showed that treatment of DES ISR with a paclitaxel-coated balloon was significantly associated with lower late lumen loss and with binary restenosis rates and MACEs than conventional BA [8]. Recently, Byrne et al. [19] reported that a paclitaxel-eluting balloon was non-inferior to repeat stenting with a paclitaxel-eluting stent in terms of diameter stenosis, binary restenosis rate, and TLR, and that both treatments were superior to conventional BA for the treatment of DES ISR.

This study has several limitations. First, small number of patients was the major limitation. Second, the lesions with ISR were not classified into DES ISR or BMS ISR lesions according to previous stent type or risk factors, especially such as diabetes, due to the limited number of patients. The limited number of patients and the heterogeneous lesions of ISR might cause no significant results of the efficacy of DES or DEB for the treatment of ISR, compared with conventional BA. Third, it involved retrospective analysis, and thus, follow-up periods varied widely. Fourth, we did not compare angiographic results, such as, late lumen loss or binary restenosis, with respect to clinical outcomes. Therefore, large-scale randomized trials are required to determine the optimal treatment and the role of DEB for coronary ISR lesions.

In conclusion, based on this small, retrospective study, DEB might be feasible and efficacious similarly to BA to DES for the treatment of coronary ISR.

\section{KEY MESSAGE}

1. This study showed that percutaneous coronary intervention using drug-eluting balloons might be a feasible alternative to conventional balloon angioplasty or drug-eluting stents implantation for treatment of coronary in-stent restenosis. Further large-scaled, randomized study assessing long-term clinical and angiographic outcomes will be needed.

\section{Conflict of interest}

No potential conflict of interest relevant to this article was reported.

\section{REFERENCES}

1. Stettler C, Wandel S, Allemann S, et al. Outcomes associated with drug-eluting and bare-metal stents: a collaborative network meta-analysis. Lancet 2007;370:937-948.

2. Wohrle J, Nusser T, Kestler HA, Kochs M, Hombach V. Comparison of the slow-release polymer-based paclitaxel-eluting Taxus-Express stent with the bare-metal Express stent for saphenous vein graft interventions. Clin Res Cardiol 2007;96:70-76.

3. Dangas GD, Claessen BE, Caixeta A, Sanidas EA, Mintz GS, Mehran R. In-stent restenosis in the drug-eluting stent era. J Am Coll Cardiol 2010;56:1897-1907.

4. Scheller B. Drug-coated balloons: the new gold standard for treatment of coronary in-stent restenosis? Cardiovasc Revasc Med 2012;13:257-259.

5. Scheller B, Hehrlein C, Bocksch W, et al. Treatment of coronary in-stent restenosis with a paclitaxel-coated balloon catheter. N Engl J Med 2006;355:2113-2124.

6. Habara S, Mitsudo K, Kadota K, et al. Effectiveness of paclitaxel-eluting balloon catheter in patients with sirolimus-eluting stent restenosis. JACC Cardiovasc Interv 2011;4:149-154.

7. Scheller B, Clever YP, Kelsch B, et al. Long-term follow-up after treatment of coronary in-stent restenosis with a paclitaxel-coated balloon catheter. JACC Cardiovasc Interv 2012;5:323-330.

8. Rittger H, Brachmann J, Sinha AM, et al. A randomized, multicenter, single-blinded trial comparing pa- 
clitaxel-coated balloon angioplasty with plain balloon angioplasty in drug-eluting stent restenosis: the PEPCAD-DES study. J Am Coll Cardiol 2012;59:1377-1382.

9. Mauri L, Hsieh WH, Massaro JM, Ho KK, D’Agostino R, Cutlip DE. Stent thrombosis in randomized clinical trials of drug-eluting stents. N Engl J Med 2007;356:1020-1029.

10. Mehran R, Dangas G, Abizaid AS, et al. Angiographic patterns of in-stent restenosis: classification and implications for long-term outcome. Circulation 1999;100:18721878.

11. Alfonso F, Zueco J, Cequier A, et al. A randomized comparison of repeat stenting with balloon angioplasty in patients with in-stent restenosis. J Am Coll Cardiol 2003;42:796-805.

12. Kastrati A, Mehilli J, von Beckerath N, et al. Sirolimus-eluting stent or paclitaxel-eluting stent vs balloon angioplasty for prevention of recurrences in patients with coronary in-stent restenosis: a randomized controlled trial. JAMA 2005;293:165-171.

13. Iofina E, Haager PK, Radke PW, et al. Sirolimus- and paclitaxel-eluting stents in comparison with balloon angioplasty for treatment of in-stent restenosis. Catheter Cardiovasc Interv 2005;64:28-34.

14. Radke PW, Kobella S, Kaiser A, et al. Treatment of in-stent restenosis using a paclitaxel-eluting stent: acute results and long-term follow-up of a matched-pair comparison with intracoronary beta-radiation therapy. Eur Heart J 2004;25:920-925.

15. Indermuehle A, Bahl R, Lansky AJ, et al. Drug-eluting balloon angioplasty for in-stent restenosis: a systematic review and meta-analysis of randomised controlled trials. Heart 2013;99:327-333.

16. Waksman R, Pakala R. Drug-eluting balloon: the comeback kid? Circ Cardiovasc Interv 2009;2:352-358.

17. Scheller B, Hehrlein C, Bocksch W, et al. Two year follow-up after treatment of coronary in-stent restenosis with a paclitaxel-coated balloon catheter. Clin Res Cardiol 2008;97:773-781.

18. Unverdorben M, Vallbracht C, Cremers B, et al. Paclitaxel-coated balloon catheter versus paclitaxel-coated stent for the treatment of coronary in-stent restenosis. Circulation 2009;119:2986-2994.

19. Byrne RA, Neumann FJ, Mehilli J, et al. Paclitaxel-eluting balloons, paclitaxel-eluting stents, and balloon angioplasty in patients with restenosis after implantation of a drug-eluting stent (ISAR-DESIRE 3): a randomised, open-label trial. Lancet 2013;381:461-467. 